Annuaire suisse de politique de développement

15 | 1996

Annuaire Suisse - Tiers Monde 1996

\title{
Enquête sur la clause sociale auprès des ONG et syndicats du Sud
}

Michel Egger et Catherine Schümperli Younossian

\section{(2) OpenEdition}

Édition électronique

URL : http://journals.openedition.org/aspd/1523

DOI : $10.4000 /$ aspd. 1523

ISSN : 1663-9669

Éditeur

Institut de hautes études internationales et du développement

\section{Édition imprimée}

Date de publication : 1 mars 1996

Pagination : 247-256

ISSN : 1660-5934

\section{Référence électronique}

Michel Egger et Catherine Schümperli Younossian, «Enquête sur la clause sociale auprès des ONG et syndicats du Sud ", Annuaire suisse de politique de développement [En ligne], 15 | 1996, mis en ligne le 16 mai 2013, consulté le 08 septembre 2020. URL : http://journals.openedition.org/aspd/1523 ; DOI : https://doi.org/10.4000/aspd.1523 


\title{
Enquête sur la clause sociale auprès des ONG et syndicats du Sud
}

\author{
Michel Egger, Catherine Schümperli
}

A l'heure de la libéralisation des échanges et de l'entrée en fonction de la nouvelle Organisation mondiale du commerce (OMC), favorisant une dérégulation et une compétition accrues, la question se pose avec acuité: faut-il introduire une clause sociale dans les échanges commerciaux internationaux?

Cette question, soulevée notamment par les Etats-Unis et la France lors de la conclusion de l'Uruguay Round du GATT en décembre 1994, est très controversée. Elle a provoqué une véritable guerre de tranchées entre, d'un côté, les pays et syndicats du Nord qui disent vouloir lutter contre le dumping social et, de l'autre, les gouvernements et certaines organisations non gouvernementales (ONG) du Sud qui voient dans cette initiative une forme déguisée de protectionnisme, visant à les dépouiller de leurs avantages comparatifs.

En Suisse, au début de 1995, quelque 70 organisations syndicales, de développement, d'environnement, de femmes et de défense des droits de l'homme ont signé une "Déclaration" sur la clause sociale, proche par son esprit de la position des grandes confédérations syndicales mondiales et européennes. Elles affirmaient ainsi la nécessité de créer certains garde-fous au sein du commerce international, et reconnaissaient la clause sociale comme un instrument de régulation sociale et de protection des droits fondamentaux de l'homme, de la femme et de l'enfant au travail.

Mais il fallait aller plus loin, approfondir cette position consensuelle, confronter le principe à la réalité, analyser les problèmes et implications concrets juridiques, politiques, économiques - d'une telle mesure. Dans cette perspective, étant donné les apparentes divergences de fond entre le Nord et le Sud, il était important de prendre plus précisément le pouls de nos partenaires, ONG et syndicats du Sud et du Nord. 
Un questionnaire centré sur la "Déclaration" suisse a donc été élaboré et une vaste consultation lancée à travers le monde. 82 organisations ont répondu, dont 67 des pays du Sud et de l'Europe de l'Est'; leurs réponses font l'objet de cet article.

\section{Type des organisations consultées}

II nous a semblé important de bien cerner qui étaient les organisations qui nous ont répondu, s'il s'agissait de syndicats ou d'organisations de développement, si elles étaient d'obédience religieuse ou laïque. Ces différents critères interviennent dans l'analyse des résultats. Notre échantillon compte: $48 \%$ d'ONG laïques, $32 \%$ d'ONG religieuses, $13 \%$ de syndicats, $7 \%$ de centres de recherches.

Les ONG représentent $80 \%$ des réponses, les syndicats $13 \%$ et les centres de recherches $7 \%$. Ces résultats s'expliquent par le fait que ce sont les oeuvres d'entraide qui ont été les principaux relais auprès de leurs partenaires dans les pays du Sud, les syndicats ayant joué le jeu moins activement.

\section{Faut-il introduire une clause sociale dans les échanges commerciaux in- ternationaux?}

Les polémiques suscitées par cette question entre le Nord et le Sud ainsi que l'attitude négative de certaines ONG du Sud laissaient présager soit des opinions très différenciées, soit une position plutôt mitigée de nos partenaires du Sud. En réalité, il n'en est rien. Prouvant une fois de plus le fossé qui peut exister entre les gouvernements et les populations à la base, les ONG et syndicats qui ont répondu à notre questionnaire se prononcent à une majorité écrasante $(91 \%)$ en faveur de la clause sociale; $6 \%$ seulement en rejettent le principe et $3 \%$ ne savent pas ou répondent de manière ambivalente.

Quels sont les motifs d'un tel plébiscite?

- Plusieurs organisations craignent que la libéralisation du commerce mondial et l'accroissement de la compétition qui en découle se fassent au détriment des droits et des conditions des travailleurs/ses, notamment du Sud, et des secteurs sociaux les plus défavorisés. Mesure de politique économique, la

1. Ces questionnaires se répartissent ainsi:

- Afrique: 16, dont 5 Burkina Faso, 7 Cameroun, 1 Madagascar, 2 Zaïre, 1 Zimbabwe;

- Amérique latine: 28, dont 2 Argentine, 2 Bolivie, 1 Brésil, 3 Costa Rica, 2 Colombie, 3 République Dominicaine, 1 Equateur, 1 Mexique, 8 Nicaragua, 1 Panama, 2 Paraguay, 1 Pérou, 1 inconnu;

- Asie: 21, dont 2 Bangladesh, 1 Hong Kong, 4 Inde, 12 Philippines, 2 Sri Lanka;

- Europe de l'Est: 2, venant de Roumanie.

Nous avons reçu en retour 15 questionnaires du Nord, dont 13 de Suisse, 1 de France et 1 d'italie. Cet article ne tient compte que des réponses en provenance du Sud ou de l'Europe de l'Est. 
clause sociale apparaît comme un filet de sécurité et un moyen de régulation nécessaire.

- La clause sociale ouvre une voie vers plus d'équité et de justice entre le Nord et le Sud, car elle contient un souci éthique le plus souvent absent des échanges commerciaux.

- La clause sociale est un instrument de garantie et de promotion des droits de l'être humain au travail, acquis ou à conquérir, dans l'industrie comme dans l'agriculture. En ce sens, elle devrait également permettre d'atténuer les effets de la politique jugée "oppressive» du Fonds monétaire international (FMI).

- La clause sociale est un moyen de fortifier l'Organisation internationale du travail (OIT) par la reconnaissance de ses conventions et l'encouragement de leur mise en œuvre.

- La clause sociale permet de renforcer les syndicats et d'améliorer les résultats des négociations collectives avec les entreprises et les gouvernements.

- La clause sociale est une condition sine qua non de la réalisation du développement durable au Nord et au Sud.

- La clause sociale est un moyen pédagogique d'amener les partenaires sociaux, notamment du Sud, à prendre conscience des implications vitales du commerce international.

Parmi tous ces partisans de la clause sociale, certains, bien sûr, relèvent le danger d'une utilisation protectionniste. Mais ce risque, qui tient plus à l'usage qu'à la nature intrinsèque de l'instrument, ne suffit pas à justifier son rejet. Simplement, sa mise en œuvre doit être rigoureusement pensée et contrôlée.

Cinq organisations (sur un total de 67) disent clairement non à la clause sociale. Les motifs invoqués par ces réfractaires recoupent les arguments classiques contre la clause sociale: risque protectionniste, mise en œuvre illusoire par manque de volonté des gouvernements, champ d'application limité aux seules industries d'exportation, difficulté à adapter les normes au niveau de développement de chaque pays, atteinte à la souveraineté nationale, etc.

\section{La clause sociale permet-elle d'atteindre les objectifs suivants?}

- favoriser le respect des droits des travailleurs/ses,

- améliorer leurs conditions de travail,

- renforcer les législations nationales sur le travail et la sécurité sociale,

- lutter contre toutes les formes d'exploitation et de dumping social,

- stimuler le sens de la responsabilité des multinationales,

- répartir plus équitablement les fruits de la croissance,

- encourager la ratification et la mise en œuvre des conventions de l'OIT,

- améliorer l'information des consommateur/trices sur les conditions de production des biens et services.

A cette question, $83,5 \%$ des ONG du Sud et des pays de l'Est répondent oui, $13,5 \%$ non et $3 \%$ ne savent pas. Le taux de réponses négatives, plus élevé 
à cette question qu'à la précédente, indique qu'un certain nombre d'organisations, en principe favorables à la clause sociale, doutent de son efficacité réelle, notamment à cause du manque de volonté politique des gouvernements. D'autres précisent l'importance de certaines conditions: plus grande mobilisation des mouvements de base, adaptation des normes aux niveaux variables de développement et aux situations différenciées sur le marché de l'emploi, pression accrue des gouvernements et des ONG du Nord au sein des instances internationales et sur certains gouvernements du Sud afin de transformer les structures économiques, politiques et sociales, internationales et nationales, qui sont souvent injustes (voir ci-dessous les mesures d'accompagnement).

\section{Faut-il, parallèlement à la clause sociale, introduire une clause écologique dans les échanges commerciaux internationaux?}

Sur cette question, on sait le fossé qui existe entre les gouvernements du Nord et ceux du Sud, qui craignent une forme d'impérialisme vert. Etant donné cette divergence assez radicale, on pouvait s'attendre à une position plutôt mitigée ou défavorable des partenaires du Sud. Curieusement, c'est tout le contraire qui s'est produit: la clause verte est plébiscitée avec 95,5\% d'opinions favorables; seuls $4,5 \%$ s'y opposent.

\section{La clause sociale doit-elle reposer sur les 7 "conventions de base" de I'OIT?}

Sur le contenu de la clause sociale, $89,6 \%$ des organisations partagent les normes minimales proposées dans la "Déclaration" suisse, liées aux conventions de l'OIT suivantes.

- Liberté syndicale et protection du droit syndical (conv. 87).

- Droit d'organisation et de négociation collective (conv. 98).

- Age minimum d'admission à l'emploi (conv. 138).

- Interdiction du travail forcé (conv. 29 et 105).

- Egalité de rémunération pour un travail de valeur égale (conv. 100) et interdiction de toute discrimination en matière d'emploi et de profession (conv. 111).

Ces sept conventions - les plus largement ratifiées par les Etats - concernent les droits fondamentaux et universels de l'être humain au travail. Dans l'optique de la "Déclaration" suisse, ce choix ne relativise pas l'importance des autres conventions sur les conditions de travail, qui doivent être améliorées prioritairement à travers les actions syndicales.

Cette précision n'a pas empêché certaines organisations de demander l'introduction de conventions complémentaires comme la 158 sur les travailleurs migrants (Costa Rica), la 110 sur les plantations agricoles (Costa Rica), la 135 sur les négociations collectives (Costa Rica, Burkina Faso), la 169 sur les peuples et tribus indigènes (Panama, Bolivie). 


\section{A quel niveau la clause sociale devrait-elle être mise en œuvre: multilaté- ral, régional, bilatéral ou privé?}

Dans leurs réponses, la plupart des organisations soulignent la nécessité de “jouer" sur les différents plans d'application pour faire avancer la question des droits des travailleurs, avec toutefois une attention particulière au niveau multilatéral.

\section{Niveau multilatéral}

Par niveau multilatéral, nous entendons une mise en œuvre dans le cadre d'enceintes internationales. $86,6 \%$ des partenaires sondés y sont favorables, $10,4 \%$ ne le souhaitent pas et $3 \%$ ne savent pas.

Deux arguments plaident pour l'introduction d'une clause sociale au plan multilatéral:

- à l'heure de la mondialisation de l'économie, le respect des droits fondamentaux des travailleurs devient un problème global qui suppose une réponse globale;

- une clause sociale permettrait de faire pression sur les gouvernements des nombreux pays qui n'ont pas encore introduit les normes minimales de travail dans leur législation nationale.

L'opposition à l'introduction d'une clause sociale au niveau multilatéral s'appuie sur deux idées clés: la domination des pays industrialisés dans les rapports de force internationaux et l'incapacité de prendre en compte, au niveau multilatéral, les préoccupations et les réalités propres à chaque région.

$86,6 \%$ des organisations sondées étant favorables à l'introduction d'une clause sociale au niveau multilatéral, il était intéressant de savoir quelle instance internationale était la plus adéquate pour son application. Quatre éléments ressortent de notre enquête:

1. Aucune organisation ne souhaite que la clause sociale soit introduite dans le cadre de l'OMC. Ce résultat confirme l'extrême méfiance des pays du Sud - gouvernements et société civile - à l'égard de la nouvelle organisation du commerce. La domination massive des pays industrialisés dans les négociations de l'Uruguay Round du GATT, les difficultés matérielles rencontrées par les pays du Sud pour suivre les débats et la crainte que l'OMC serve surtout les intérêts des multinationales expliquent sans doute ce rejet.

2. En revanche, $49 \%$ des organisations souhaitent la création d'une articulation entre I'OIT et l'OMC. Motif? les deux organisations ont une certaine expérience dans les domaines concernés (normes de travail et commerce), mais l'OIT seule ne pourra pas mettre en œuvre la clause sociale - "elle a démontré son manque de pouvoir" - et l'OMC n'y est pas intéressée risque d'entraver la liberté des échanges. D'où la nécessité de créer un organisme conjoint efficace. Quelle forme pourrait prendre ce dernier? Les propositions des partenaires sont de trois ordres: 
- L'OIT serait chargée de surveiller la mise en œuvre de la clause sociale et jouerait le rôle d'instance de recours; I'OMC prendrait la responsabilité de l'application d'éventuelles sanctions et de la création d'un fonds pour des mesures d'accompagnement. Sur la manière de coordonner les deux organisations, certaines ONG suggèrent la constitution d'un secrétariat permanent avec des représentants de l'OMC et de I'OIT, d'autres une commission internationale ad hoc avec des représentants régionaux qui réceptionnent et canalisent les dénonciations. Dans tous les cas, comme le souligne un partenaire du Panama, il est nécessaire de renforcer le système de contrôle de l'OIT, via, par exemple, le conditionnement de son aide technique au respect des conventions.

- La création, au sein de l'OMC et sous la responsabilité de l'OIT, d'un organe permanent chargé d'analyser la dimension sociale dans la libéralisation des échanges.

- La mise sur pied d'un forum où les deux organisations se réuniraient régulièrement pour débattre et garantir un niveau acceptable au double plan du rendement économique et des conditions de travail.

3. Un quart (25\%) des organisations souhaitent que la question de la clause sociale soit traitée uniquement dans le cadre de l'OIT; l'ancienneté, l'expérience et le système tripartite de l'institution plaident en sa faveur. Cependant, la plupart reconnaissent son inefficacité et insistent sur les réformes nécessaires pour rendre ses décisions plus contraignantes: contrôles accrus sur le terrain, conditionnalité de l'aide technique, etc.

Ce faible taux de suffrages en faveur de l'OIT révèle bien le scepticisme des ONG à son égard, certaines allant même jusqu'à stigmatiser son «inefficacité". Dans une publication, "Des valeurs à défendre, des changements à entreprendre", Michel Hansenne, directeur général de I'OIT, relève bien le problème de son organisation: "La question majeure est de savoir si l'OIT peut maintenir, dans un cadre volontaire, le cercle vertueux d'une "émulation" en faveur du progrès social dans une situation où mondialisation de l'économie et renforcement de la concurrence conjuguent leurs effets dans le sens contraire” (p. 58). Aujourd'hui, l'OIT se trouve tiraillée entre deux nécessités contradictoires: d'un côté, maintenir la "démarche volontariste" de dialogue et de coopération qu'elle a choisie pour promouvoir le progrès social, et à laquelle elle ne saurait renoncer sans risquer de perdre la confiance de ses membres; de l'autre, augmenter l'efficacité de son action normative en tant que régulateur du commerce international. Tant que l'organisation n'aura pas décidé clairement le rôle qu'elle entend jouer sur la scène de l'économie mondiale, elle aura du mal à susciter la pleine adhésion des différents acteurs.

4. $21 \%$ des organisations - visiblement déçues des instances internationales existantes - souhaitent la création d'une nouvelle institution, tout en reconnaissant l'extrême difficulté à réaliser un tel projet. 


\section{Niveau régional}

Les traités régionaux ${ }^{2}$ sont-ils un lieu adéquat pour l'introduction de normes sociales dans les accords commerciaux? $74,6 \%$ des organisations le pensent, $3 \%$ est contre et $22,4 \%$ ne savent pas. Le soutien à cette proposition repose sur le fait que chaque bloc économique peut définir des critères adaptés au développement socioéconomique de la région. Comme le souligne une ONG du Cameroun, «les habitants d'une même aire géographique partagent une culture, des problèmes et des intérêts semblables."

\section{Niveau bilatéral}

Seules $62,6 \%$ des organisations sont favorables à une clause sociale dans les accords commerciaux entre deux pays, $19,4 \%$ sont contre et $18 \%$ ne savent pas. Cette réserve s'explique par l'inégalité des relations économiques entre pays industrialisés et pays en développement et par le risque de mesures protectionnistes. Comme le relève une ONG indienne: "Le danger du niveau bilatéral, c'est que les pays industrialisés peuvent prendre des mesures discriminatoires - voire user de représailles - contre un pays en développement, en dépit ou à l'encontre de la réalité objective des faits."

\section{Niveau privé}

Le niveau privé renvoie à des initiatives visant à améliorer les conditions de travail, comme les codes de conduite des multinationales ou les labels dans le domaine du "commerce équitable", qui sont souvent plus faciles à réaliser que les décisions entre Etats et touchent plus directement les acteurs concernés (entreprises, producteurs, consommateurs).

Les organisations sondées sont plutôt favorables (à $68,6 \%$ ) à une clause sociale sous cette forme; $4,5 \%$ sont contre et $26,9 \%$ ne savent pas. Les codes de conduite sont, d'une manière générale, perçus comme un moyen de responsabiliser les entreprises et de les obliger à adopter des comportements éthiques dans les pays où elles investissent et d'où elles retirent des bénéfices.

2. Les principaux accords économiques régionaux sont les suivants: Union européenne (UE), Association des Nations de l'Asie du Sud-Est (ASEAN), Coopération économique de l'Asie-Pacifique (APEC), Accord de libre-échange nord-américain (ALENA), Marché commun de l'Amérique centrale (MCAC), Groupe andin, Marché commun du Cône Sud (MERCOSUR), Union économique et monétaire de l'Ouest africain (UEMOA), Union du Maghreb arabe (UMA), Communauté économique des Etats de I'Afrique de l'Ouest (CEDEAO), Union douanière et économique de l'Afrique centrale (UDEAC), Union douanière de l'Afrique australe (SACU), Communauté de développement de l'Afrique australe (SADC), Groupe de Visegrad, Communauté des Etats indépendants (CEI). Source: Courrier de la planète, Intégration régionale, le jeu des blocs, $n^{2}$ 28, mai-juin 1995. 
Cette attitude positive n'empêche cependant pas l'expression d'une méfiance à l'égard des multinationales, qui "bien souvent font pression pour affaiblir les législations en matière de droit du travail».

Pour rendre contraignant le respect des droits fondamentaux des travailleurs/ses, la clause sociale peut-elle être accompagnée de sanctions?

Actuellement, sanctionner un Etat souverain en lui interdisant l'accès à certains marchés constitue une mesure d'exception en droit international. Malgré cela, $90 \%$ des organisations sondées reconnaissent la nécessité d'imposer des sanctions pour faire respecter la clause sociale au niveau multilatéral et $64 \%$ acceptent l'idée de conditionner l'aide publique au développement en cas de violation des standards sociaux inclus dans un accord commercial bilatéral.

Parmi les différents instruments coercitifs possibles, la préférence (55\%) va clairement à une sanction ciblée sur les produits incriminés (interdiction de l'accès au marché), mesure à l'évidence la plus appropriée et certainement la moins discriminatoire pour un pays. Un autre outil intéressant les organismes consultés sont les préférences douanières, qui peuvent être utilisées soit positivement au sens d'un octroi (48\%), soit négativement par la suppression de tarifs préférentiels (43\%). En revanche, les organisations sont beaucoup plus réservées envers les mesures plus contraignantes pour l'ensemble d'un pays, comme l'augmentation générale des tarifs douaniers par exemple.

D'une manière générale, syndicats et ONG insistent sur la nécessité d'apporter un appui technique aux pays en développement et sur le fait que l'aide publique au développement doit garder un caractère apolitique et non-discriminatoire. Cela dit, ils relèvent aussi l'impossibilité d'un véritable développement quand les droits des travailleurs/ses sont bafoués et quand certains seulement bénéficient de leurs efforts. Dans ces conditions, $64 \%$ acceptent l'idée de conditionner l'aide au développement, pour autant que les ONG n'en fassent pas les frais et que seule l'aide intergouvernementale soit affectée.

Fataliste ou lucide, une ONG du Panama relève: "Le développement de nos pays pauvres est de toute manière conditionné d'un point de vue économique, politique, social, culturel et technologique, pour ne pas dire imposé par le jeu des puissances mondiales. Si la question de savoir qui va décider de la conditionnalité de l'aide nous préoccupe, nous n'en sommes pas moins convaincus de la nécessité du respect de normes minimales en matière de travail. Une claire définition des critères et des responsabilités devrait permettre d'éviter que la conditionnalité de l'aide se transforme en arme économique et politique."

\section{Mesures d'accompagnement}

Conscients de la difficulté d'introduire une clause sociale uniforme dans un environnement international inégalitaire, nous avons demandé à nos partenaires quelles étaient les mesures d'accompagnement à mettre en œuvre pour réaliser les objectifs de la clause sociale dans leur pays. 
Trois mesures ressortent nettement du lot: la nécessité d'une redéfinition des programmes d'ajustement structurel du Fonds monétaire international, le soutien aux mouvements de base et syndicats, les transferts de technologies et les mesures de désendettement. En revanche - surprise de taille - les transferts financiers par le biais de l'aide publique au développement arrivent en dernière position.

\section{Quel doit être le rôle des ONG et des syndicats, du Sud et du Nord, dans l'élaboration et la mise en œuvre de la clause sociale?}

Sur cette question, les partenaires du Sud ont été très prolixes. D'une manière générale, les ONG et syndicats du Sud et du Nord devraient:

- participer activement à l'élaboration, l'application et le contrôle de la clause sociale, en collaborant plus étroitement avec l'OIT et en dénonçant les abus et violations éventuels des gouvernements et des entreprises;

- faire du lobbying pour rendre l'OMC plus transparente et démocratique;

- lutter contre les gouvernements et les compagnies qui ignorent les critères de la clause sociale ou ne les respectent pas;

- veiller à ce que les intérêts de la base et des travailleurs/ses soient sauvegardés;

- développer le dialogue et la collaboration entre ONG de développement et syndicats, à l'intérieur d'un même pays, mais aussi entre le Nord et le Sud. Cela à travers, par exemple, la constitution d'un réseau d'information, le lancement de campagnes communes, des actions de soutien mutuel, la définition d'objectifs communs et de positions conjointes.

\section{Conclusion}

En résumé ${ }^{3}$, les ONG et syndicats du Sud et des pays de l'Est qui ont répondu à notre sondage disent majoritairement oui à l'introduction d'une clause sociale dans les échanges commerciaux internationaux. Ils la considèrent comme une mesure efficace pour garantir le respect des droits fondamentaux des travailleurs/ses et améliorer les conditions de travail. Pour une majorité - souvent très large - une telle clause sociale, indissociable d'une clause écologique et fondée principalement sur sept conventions-clés de l'OIT, doit être mise en œuvre à tous les niveaux - multilatéral, régional, bilatéral et privé; un accent particulier porte cependant sur le plan multilatéral, notamment à travers la création d'une organisation conjointe OIT/OMC. Afin de donner à la clause

3. Un rapport détaillé et exhaustif incluant les prises de position des ONG et syndicats du Nord, mais aussi des tableaux, des citations, le texte de la "Déclaration" et le questionnaire, peut être commandé à la Déclaration de Berne, CP 212, 1000 Lausanne 9, tél. 021/624 5417 - Fax. 021/ 62454 19, ou à Pain pour le prochain, 3, Chemin du Boisy, 1004 Lausanne, tél. 021/646 3077 - Fax. 021/647 6707. 
sociale une certaine réalité et efficacité, la plupart des organisations reconnaissent la nécessité de sanctions comme l'interdiction de l'accès au marché de produits incriminés et la conditionnalité de l'aide.

Expression des mouvements de base, ces résultats se démarquent des prises de position des gouvernements du Sud, de certaines grosses ONG du Sud et de certains mouvements tiers-mondistes européens.

Au-delà de ses enseignements, cette vaste consultation a permis de susciter des processus de réflexion très vivants sur la question de la clause sociale, aussi bien dans les ONG et syndicats du Sud que dans ceux du Nord. 